Bangladesh Journal of Anatomy July 2013, Vol. 11, No. 2 pp. 68-70

\title{
Variations in the Location of Basilar Artery on Ventral Surface of Pons
}

\author{
Anjuman Ara Sultana ${ }^{1}$, Shamim Ara ${ }^{2}$, Mushfika Rahman ${ }^{3}$, Halima Afroz ${ }^{4}$, Rahanur Akter $^{5}$, \\ Farhana Akter ${ }^{6}$, Kanij Fatema ${ }^{7}$
}

\begin{abstract}
Context: Basilar artery is formed by the union of right \& left vertebral arteries at the lower border of pons / mid medullary level which courses upward along 'Basilar groove' on the anterior surface of pons \& supplies the anatomical structures within the posterior cranial fossa of brain. The Circle of Willis is the principal arterial anastomotic trunk of brain formed by anastomosis between the branches of internal carotid arteries and terminal branch of basilar artery. It is responsible for collateral circulation of brain, specially of old people - who have reduced blood supply to brain due to senile arteriosclerosis. Cerebrovascular diseases due to basilar artery is more common in men than women. Anatomy of basilar artery and its branches are very complex and variable. So, detailed morphogical knowledge is essential for proper diagnosis and treatment of neurological disorder. To identify whether there is any variations in the location of basilar artery in relation to basilar groove on the ventral surface of pons.
\end{abstract}

Materials and Methods: A cross-sectional descriptive type of study was carried out in the Department of Anatomy, Dhaka Medical College, Dhaka, from January 2010 to December 2010. Seventy(70) postmortem basilar arteries were collected from unclaimed dead bodies that were under examination in the morgue of Department of Forensic medicine, Dhaka Medical College Dhaka. The study was approved by the Ethical Review Committee (ERC) of Dhaka Medical College. The position of basilar artery in all the samples were either in the midline, right to midline or left to midline. Formalin fixed brain was washed with plain tap water to remove excess formalin and was kept on metallic tray. Location of basilar artery was examined carefully by hand magnifying glass and noted.

Results: In the present study, location of basilar artery was found at midline in $70 \%$ brain, right to midline in $17 \%$, left to midline in $13 \%$.

Conclusion: Further studies in large scale for preparing a database of anatomy of basilar artery are recommended.

Key Words: Location, basilar artery, pons.

\section{Introduction}

Basilar artery is one of the main arteries that supplies the posterior portion of brain, the area

1. Assistant Professor. Department of Anatomy, Shaheed Suhrawardy Medical College.

2. Professor \& Head, Department of Anatomy, Dhaka Medical College, Dhaka-1000.

3. Associate Professor, Department of Anatomy, Dr. Sirajul Islam Medical College, Dhaka.

4. Associate Professor. Department of Anatomy, Green Life Medical College, Dhaka-1205.

5. Lecturer, Department of Anatomy, Shaheed Suhrawardy Medical College.

6. Assistant Professor, Department of Anatomy, Dhaka National Medical College, Dhaka.

7. Assistant Professor, Department of Anatomy, Jahurul Islam Medical College.

Correspondence : Dr. Anjuman Ara Sultana supplied by basilar artery is also called posterior cerebral circulation ${ }^{1,2}$. Basilar artery is the most important artery in the posterior circulation ${ }^{3}$. Basilar artery is formed by the union of right and left vertebral arteries at the lower border of pons or mid medullary level ${ }^{1,4}$ \& 5 . Then it courses upward along 'basilar groove' on the anterior surface of the pons, and it ends at the upper border of pons by bifurcating into two posterior cerebral arteries ${ }^{6}$. The posterior cerebral artery, branch of basilar artery completes an arterial polygon called the 'circle of Willis', which is the principal arterial anastomotic trunk of brain ${ }^{1}$. Normally little blood flows around this circle, if one major vessel occludes the communicating arteries may allow 
critically important anastomotic flow and prevent neurological damage ${ }^{7}$. The most frequent and severe atherosclerotic (degenerative proliferative) change occur in upper and lower part of basilar artery and the first part of the posterior cerebral artery. Severe narrowing of vessels sufficient to cause vascular insufficiency occurred in $2 \%$ of case as age $30-40$ years and in as many as $6-8 \%$ in patient from age 60-70 years ${ }^{1}$. Anatomical variation occasionally occurs in basilar artery and its branch. Most frequent anatomical variation of basilar artery is short segments of duplication or island formation. The superior cerebral artery may be multiple ${ }^{8}$. The proximal and mid basilar arteries are the most common sites of stenosis and most often cause of infarction in pons ${ }^{9}$. Aneurysm often develops at the site of branching of basilar artery and near the arterial circle and they can rupture or leak causing subarachnoid hemorrhage ${ }^{10}$. Cerebrovascular disease was found $22 \%$ in the year $2004^{11}$ in Mymensingh Medical College Hospital. Stroke due to basilar artery comprises approximately $10 \%$ to $15 \%$ of all stroke, more common in man than woman. Structures commonly affected by basilar artery infarction are brain stem $(60 \%)$, cerebellum (50\%). Basilar artery related clinical condition such as posterior circulation stroke, migraine, aneurysm, atherosclerotic changes are very common throughout the world. Anatomy of the basilar artery and its branches in Bangladeshi people are necessary for neurosurgeon, neurologist for proper diagnosis and treatment of cerebrovascular disease, aneurysm, tumour, epilepsy, migraine and other form of vascular anomalies.

\section{Materials \& Methods}

\section{Materials}

The present study was performed on 70 post mortem basilar arteries collected from unclaimed dead bodies between 16-60 years of age that were under examination in the morgue of Department of Forensic Medicine, Dhaka Medical College, Dhaka, Dhaka from January 2010 to. December 2010. The study was approved by the Ethical Review Committee (ERC) of Dhaka Medical College. After legal formalities and requisite permission from the Department of Forensic Medicine. The whole brains were collected within 24-36 hours of death. Soon after collection, each sample was gently washed with tap water on a dissection tray. Blood and blood clots were removed. After collection of whole brain, $100 \mathrm{ml}$ of $40 \%$ formaldehyde solution was injected by using a 50cc syringe into the brain through the surfaces (superolateral and inferior surfaces). Then it was preserved in $40 \%$ formaldehyde solution for 15 days. After 15 days the present study was made with these fixed specimens.

\section{Methods}

Place and duration of study:

This study was carried out in the Department of Anatomy, Dhaka Medical College, Dhaka, Bangladesh, from January 2010 to December 2010. During collection of the samples appropriate age and the cause of death were noted from morgue's record book and the samples were tagged bearing code numbers for subsequent identification.

\section{Observation of location of basilar artery on ventral surface of pons}

Formalin fixed brain was washed with plain tap water to remove excess formalin and was kept on metallic tray. Location of basilar artery on the ventral surface of pons was examined carefully by hand magnifying glass and noted.

\section{Ethical Clearance}

This study was approved by this Ethical Review Committee of Dhaka Medical College, Dhaka.

\section{Results}

Results are shown in the Table -I, Fig-1

Table-I

Location of basilar artery on sulcus basilaries.

\begin{tabular}{lcc}
\hline At midline & $\begin{array}{c}\text { Right to midline } \\
\text { No. }(\%)\end{array}$ & $\begin{array}{c}\text { Left to midline } \\
\text { No. }(\%)\end{array}$ \\
\hline $49(70 \%)$ & $12(17 \%)$ & $9(13 \%)$ \\
\hline
\end{tabular}

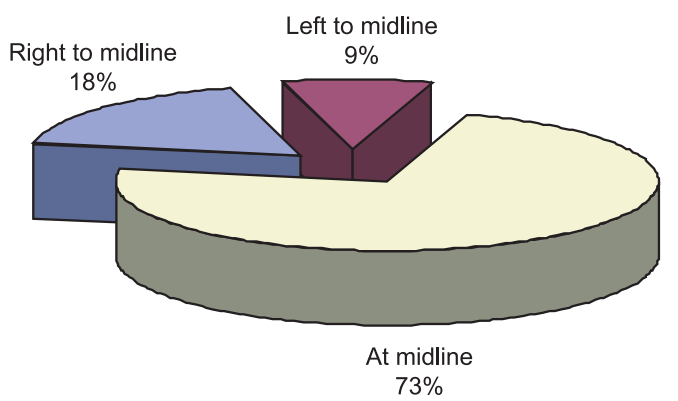

Fig.-1: Location of basilar artery on sulcus basilaris 


\section{Discussion}

In the present study, location of basilar artery at sulcus basilaris was found at midline $70 \%$, right to midline $17 \%$, left to midline $13 \%$. Rogers ${ }^{12}$ Kiernan ${ }^{13}$, Parena ${ }^{14}$, Nolte ${ }^{7}$, Pai et al. ${ }^{6}$, Snell ${ }^{5}$, Crossman ${ }^{4}$ reported basilar artery located in mid line of pons, which was similar to the present study. All these studies are similar regarding the location of basilar artery on ventral surface of pons. But more specific locations of basilar artery, such as right or left to midline could not be compared, as other studies were not available regarding these locations.

\section{Conclusion}

In present study, commonest location of basilar artery at sulcus basilaris was found at midline $70 \%$ (49), right to midline $17 \%$ (12), left to midline $13 \%$ (9). Further studies to find out the cause and functional / clinical correlations of this variation are recommended.

\section{References}

1. Chusid JG.Diorder due to vascular disease of the central nervous system.Correlative neuroanatomy \& functional neurology. USA. Lange Medical Publications 1985: 338-46

2. Bartomirt and Bogguslasky. Vascular disorder of the posterior circulation- an anatomicoclinical overview. Acenr. 2004; 4(2): 7-9.

3. Flores SC. and Muengtaweepongsa S. Basilar artery- Thromboses.2009; available from: http/ /emedicine.medscape.com/articel/1161044overview.

4. Crossman AR. ed. Neuroanatomy. In: Standring S, Ellis H, Heally JC, Johnson D, Williams A, Collins P, et al. eds. Gray's Anatomy: The anatomical basis of clinical practice. $39^{\text {th }}$ ed. Edinburgh: Elsevier Churchill Livingstone 2005: 298-301.
5. Snell RS. The blood supply of the brain and spinal cord. in: Clinical Neuroanatony for medical student. $8^{\text {th }}$ ed. Philadelphia: Lippincott Williams and Wilkins 2008: 469-97.

6. Pai BS, Varma RG, Kulkarni RN, Nirmala S, L. Microsurgical anatomy of posterior circulation. Neurol India 2007; 55(1): 361-41.

7. Nolte J. Blood supply of the brain. In: The human Brain. $5^{\text {th }}$ ed. St. Louis: Mosby 2002: 119-46.

8. Berkovitz BKB, Moxham BJ. A textbook of head and neck anatomy. $1^{\text {st }}$ ed. Barcelona: Wolfe Publishing Ltd. 1988: 577-80.

9. Woolfenden A, Tong D, Norbash A, Ali A, et al. Basilar artery stenosis- clinical and neuroradiographic feature. Journal of stroke and cerebrovaseular disease 2009; 9(2): 57-63.

10. Tulleken C A F. and Luiten L F B. The basilar artery bifurcation: Microscopical anatomy. Acta Neurochir. 2005;85:50-55.

11. Ahmed S, Hossain S, Rahman MK. Disease Pattern at Neurology Outpatient Department of Mymensingh Medical College Hospital, Mymensingh-One Year Study in the Year 2002.Bangladesh Journal of Neuroscience 2006; 22(2): 89-92.

12. Rogers AW, Jacob $S$. The blood supply of head and neck. A textbook of anatomy. $1^{\text {st }}$ ed. Edinburgh: Churchill Livingstone 1992. 597-99.

13. Kiernan JA. Blood supply of the central nervous system. In: Barr's the human nervous system: an anatomical viewpoint. $7^{\text {th }}$ ed. Philadelphia: Lippincott-Raven 1998: 801-11.

14. Parenea. Blood supply of the central nervous system. Carpenter's human neuro anatomy. $9^{\text {th }}$ edition USA: Williams \& Wilkins 1996: 113-17. 